RUNNING HEAD: Timing of emergence of the other-race effect

\title{
In Infancy the Timing of Emergence of the Other-Race Effect is Dependent on Face Gender
}

\author{
Diana Su Yun Tham ${ }^{1}$ \\ J. Gavin Bremner ${ }^{1}$ \\ Dennis Hay ${ }^{1}$ \\ ${ }^{1}$ Lancaster University
}

INFANT BEHAVIOR AND DEVELOPMENT (IN PRESS)

Correspondence: Dr. Diana Su Yun Tham, Department of Psychology, Lancaster University, Lancaster, LA14YF, d.tham@lancaster.ac.uk 


\begin{abstract}
Poorer recognition of other-race faces relative to own-race faces is well documented from late infancy to adulthood. Research has revealed an increase in the other race effect (ORE) during the first year of life, but there is some disagreement regarding the age at which it emerges. Using cropped faces to eliminate discrimination based on external features, visual paired comparison and spontaneous visual preference measures were used to investigate the relationship between ORE and face gender at 34 and 8-9 months. Caucasian-White 3- to 4-month-olds' discrimination of Chinese, Malay, and Caucasian-White faces showed an own-race advantage for female faces alone whereas at 8-9 months the own-race advantage was general across gender. This developmental effect is accompanied by a preference for female over male faces at 4 months and no gender preference at 9 months. The pattern of recognition advantage and preference suggests that there is a shift from a female-based own-race recognition advantage to a general own-race recognition advantage, in keeping with a visual and social experience-based account of ORE.
\end{abstract}

Keywords: Race, Gender, Infancy, Face Recognition 


\section{Introduction}

Faces are perhaps one of the most important categories of visual stimuli in our environment. However, recognition ability is far from uniform across faces, being subject to variation across races in particular. The consistent finding that adults show better recognition for own-race than other-race faces is referred to as the other-race effect (ORE; see Meissner \& Brigham, 2001 for a review). One of the most influential theories explaining the ORE and other face processing advantages is Valentine's (1991) multidimensional face-space (MDS) model. According to it, familiarity with a given face type (e.g., own-race) provides the opportunity to learn to differentiate between exemplars within this familiar category (Quinn \& Tanaka, 2007), whereas such within-category discrimination is not possible for unfamiliar face types (e.g., other-race). Thus, the ORE is assumed to be a consequence of predominant experience with faces of individuals' own-race.

However, this effect is believed to be initially absent in infants, developing during the first year of life. This is consistent with the notion of 'perceptual narrowing' (Nelson, 2001). Specifically, infants begin life with a face perception system with a broad tuning that allows the processing of faces in general. Then, as they experience more faces, the face system narrows to specialize for the type(s) of face that they encounter most (Bar-Haim, Ziv, Lamy, \& Hodes, 2006; Kelly, Liu, et al., 2007a; Kelly et al., 2009; Kelly, Quinn, et al., 2007b; Quinn et al., 2008; Quinn, Yahr, Kuhn, Slater, \& Pascalis, 2002).

There is increasing evidence that infants' face processing is strongly influenced by their visual and social environment. Work has shown that spontaneous preference and enhanced discrimination of a specific category (i.e., own-race, female gender) is not present at birth but emerges during the early months (Kelly et al., 2005, 
2007a; Quinn et al., 2008). For example, 3-month-olds show a visual preference for both the gender (Quinn et al., 2002; Quinn et al., 2008) and race (Kelly et al., 2005) of their primary caregiver. In contrast, there is inconsistent evidence regarding age of onset for own-race recognition advantage. Although investigators generally agree that the ORE develops within the first year, some studies found that 3-month-olds showed an own-race recognition advantage (Barrera \& Maurer, 1981; Hayden et al., 2007; Sangrigoli \& de Schonen, 2004), whereas others (Ferguson, Kulkofsky, Cashon, \& Casasola, 2009; Kelly et al., 2009; Kelly, et al., 2007b) found ORE developing between 6 and 9 months. The different findings of these studies could be explained by two differences in the stimuli used. Firstly, studies that demonstrated broad recognition ability at 3 months and an onset of ORE between 6 and 9 months presented faces with external information (Ferguson et al., 2009; Kelly et al., 2007b; 2009) whereas studies that found an onset of ORE at 3 months presented faces without external information (Hayden et al., 2007; Sangrigoli \& de Schonen, 2004). There is evidence that very young infants tend to process the external features of faces (low-level sensory cues) more easily than their internal details (Bartrip, Morton, \& de Schonen, 2001; Hainline, 1978; Haith, Bergman, \& Moore, 1977; Maurer \& Salapatek, 1976; Pascalis, de Schonen, Morton, Deruelle, \& Fabre-Grenet, 1995; Rose, Jankowski, \& Feldman, 2008; Simpson, Jakobsen, Fragaszy, Okada, \& Frick, 2014; Turati, Cassia, Simion, \& Leo, 2006). Young infants are less likely than older ones to scan the inner features of faces (Maurer \& Salapatek, 1976; Turati et al., 2006) and a shift away from the tendency to use external facial information is found between 5 and 9 months (Rose et al., 2008). Therefore, the inclusion of external facial information in both Ferguson et al. (2009) and Kelly et al. (2007b, 2009) may have permitted face identification on the basis of low-level external contour cues. It can be 
argued (e.g., Rose et al., 2008; Turati et al., 2006) that the ORE is linked to higherlevel configurational coding of faces and so its absence in 3-month-olds when external information was presented may have been due to their tendency to rely on external cues when these are available. The findings from these studies of a developing ORE in older infants may be due to the shift to configurational processing more than the development of ORE. And the possibility remains that other studies revealed an ORE in 3-month-olds because infants were compelled to process internal configuration.

Secondly, Hayden et al. (2007) and Sangrigoli and de Schonen (2004), who showed the ORE in infancy at 3 months, presented only female faces whereas Kelly et al. (2007b, 2009) used both female and male faces. Because 3-month-old infants show a preference and processing advantage for female faces when their primary caregiver is female (Quinn et al., 2002, 2008), it is possible that the own-race recognition advantage develops first for female faces and later for male faces when their primary caregiver is female. For example, newborns can discriminate their mother's face from another female face (Bushnell, Sai, \& Mullin, 1989; Pascalis, et al., 1995; Walton, Bower, \& Bower, 1992), two unfamiliar female faces when external features are available (Turati et al., 2006), and by 3 months, two unfamiliar female faces when external features are not available (Hayden et al., 2007; Sangrigoli \& de Schonen, 2004). However, they do not have the same discrimination abilities when it comes to their father's face. Newborns (Walton et al., 1992) and 4-month-olds (Ward, 1998) are not able to discriminate their father's face from another unfamiliar male face. Even by the age of 7 months, infants still have difficulty discriminating among unfamiliar male faces (Fagan, 1976). This may be related to the developmental task of forming an attachment relationship in infancy (e.g., Scherf \& Scott, 2012) whereby 
the need to perceptually discriminate primary caregiver(s) from all other individuals induces the emergence of recognition advantages for faces of the same race, gender, and age of the primary caregiver. Note that the absence of face gender differences at 3 months in work by Kelly et al. (2007b; 2009) could be because 3-month-old infants were able to rely on external facial information, a level of identification at which an experience-based female face advantage might not emerge.

The aim of the work that we report here is to investigate the possible interaction between face gender and face race in the emergence of the ORE. Mindful of the considerations above, we presented faces on a black background and cropped out the overall shape of the head. This step was taken to ensure that infants were not using hairstyle/hair shape cues; forcing them to process more subtle cues and so providing circumstances under which we would expect to see evidence of face specialization if it has occurred. Secondly, focusing on knowledge that young infants show a processing advantage for female faces that is likely related to the characteristics of their primary caregiver (Quinn, et al., 2002, Scherf \& Scott, 2012), we specifically investigated infants with female (own-race) primary caregivers. We used two measures to assess face recognition and face preference of 3- to 4-month-old and 8- to 9-month-old Caucasian-White infants, recognition through the visual paired comparison (VPC) procedure used by Kelly et al. $(2007,2009)$ and preference through the visual preference (VP) procedure used by Quinn et al $(2002,2008)$.

On the basis of a caregiver-primacy model in which young infants primarily experience and form their principal attachment to female caregivers, our prediction was that young infants would be better able to recognize own-race female faces and prefer them in comparison with own-race male faces and other-race faces, but that there would be no such effects in the case of male faces. On the other hand, we 
expected that older infants' cumulative experience extending the effect beyond the primary caregiver would result in a processing advantage for own race faces that is applied to male as well as female faces.

\section{Experiment 1}

In Experiment 1, we investigated the relation between face gender and the ORE. Three- to 4-month-old and 8- to 9-month-old Caucasian-White infants were presented with a VPC test similar to the one by Kelly et al $(2007,2009)$ but with cropped female and male Caucasian-White, Chinese, and Malay faces. If the cropped faces meant that specialization could occur for most experience faces, we expected 3to 4-month-olds to show a face recognition advantage for female own-race faces.

\section{Method}

Participants. Seventy-five Caucasian-White babies from the United Kingdom, 37 aged 3-4 months ( 25 females, mean age $=123$ days: range $104-140$ days) and 38 aged 8-9 months (15 females, mean age 276 days: range 259 - 293 days) were tested. All participants were healthy full term infants recruited from the maternity unit of the Royal Lancaster Infirmary. Eighteen babies were excluded from the final analysis due to fussiness $(n=9)$, side bias during testing $(>95 \%$ looking time to one side; $n=5)$ and equipment failure $(n=4)$. A further 5 infants who were directly exposed to other-race faces (e.g., family friend, neighbours) were also removed. The population of Lancaster is predominantly Caucasian-White, so any form of exposure to other race faces is likely to be very low. All parents reported female own-race face type as infants' primary caregiver. Participants were randomly assigned to one of the three ethnic conditions, with 12 to 13 in each condition. 
Apparatus. Testing sessions were conducted in a small, dimly lit infant testing laboratory in the infancy laboratory, Lancaster University. Infants sat on a parent's lap $60 \mathrm{~cm}$ from a Panasonic (32 inch) colour display monitor, placed at infants' eye level. A video camera positioned in front of the display monitor oriented towards the infant fed to another monitor in an adjoining control room to allow the experimenter to record infants' looking times. Habit X 1.0 software (Cohen, Atkinson, \& Chaput, 2004) running on a Macintosh computer was used to control the presentation of stimuli, record infants' looking times during each trial, and calculated when infants met the habituation criterion.

Stimuli. The images were photographs of students (age 18 to 30 years) from Lancaster University, UK (Caucasian-White faces), and Sunway University, Malaysia (Chinese and Malay faces) database. Both other-race faces had different skin tone from Caucasian-White faces and Chinese faces had pronounced featural differences. The faces were aligned on eye and mouth positions and luminance normalized for all images $($ mean $=110)$. These faces $(30$ of each face race) were selected based on 20 adult Caucasian-White and 20 adult Chinese raters' judgments on a 7-point scale of clarity, face typicality, and attractiveness. Twelve adult female and male faces with the highest scores in clarity and face typicality, but average scores on attractiveness, were selected as stimuli for this experiment. The latter criterion was applied to ensure that none of the faces was distinctively attractive which could result in spontaneous preference for attractiveness (Slater et al., 1998). The faces were from three different ethnic groups (4 Chinese, 4 Malays, and 4 Caucasian-Whites). Each was photographed in two views (frontal view and a $3 / 4$ profile view) leading to 24 images in total. The varied face view between familiarisation and testing is preferred to using identical pictures because it ensures that face recognition - as opposed to picture 
recognition (i.e., image matching) - is tested (Bruce \& Young, 1986). All pictures were color portraits, cropped to the same oval shape with little hair information, and were approximately the same quality (e.g., on a black background, equal size, same eye and hair color).

Procedure. Participants were randomly assigned to one of the three ethnicgroup conditions (Chinese, Malay, and Caucasian-White) and one of the two vieworder conditions (frontal-profile or profile-frontal, see Figure 1). Infants were habituated and tested with male and female face blocks with a 10 min break between counterbalanced blocks. For example, following habituation to female CaucasianWhite faces in the frontal orientation, infants were tested with the familiar stimulus paired with a novel female Caucasian-White stimulus, both displayed in the same 3/4 profile orientation. Face recognition was indicated by longer looking at the novel face. We varied face views between habituation and test phases to ensure that face recognition was tested as opposed to picture recognition (Bruce \& Young, 1986; Bruce et al., 1999).

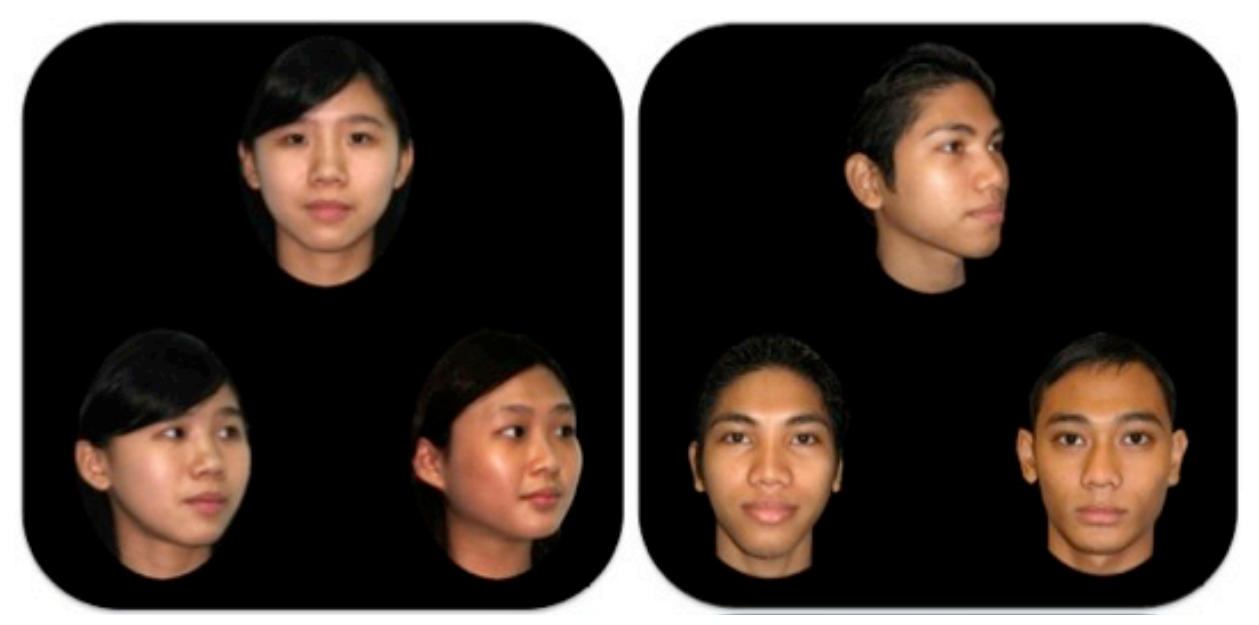

Figure 1. Sample stimuli from the Chinese female and Malay male conditions. The habituation face is the face on top while the test faces (novel and familiar) are shown below. 
Habituation Phase. Each trial consisted of presentation of a single face measuring $15 \mathrm{~cm} \times 18 \mathrm{~cm}\left(14^{\circ}\right.$ visual angle horizontally and $17^{\circ}$ visual angle vertically) on a $45 \mathrm{~cm} \times 30 \mathrm{~cm}\left(40^{\circ}\right.$ visual angle horizontally and $28^{\circ}$ visual angle vertically) monitor. Although this face differed between infants, for each infant the same face was presented across habituation trials. Half the infants were habituated to a frontal view and half to a profile view. The habituation session began with the presentation of an attention-getter, an animated oscillating sounding rattle presented prior to each trial to direct the infant's gaze toward the monitor. Habituation trials began once infants attended to the monitor. Each trial continued until 30 seconds had elapsed, or when infants looked away for two continuous seconds once they had looked for a minimum of 1 second. The experimenter, who was blind to the stimulus presented, recorded infants' looking times on each trial using Habit X 1.0 (Cohen et al., 2004) by pressing a key while the infant fixated the image. When the infant averted gaze from the screen for two continuous seconds, the trial ended and the image disappeared. The attention-getter was then repeated to get the infant's gaze back to the screen whereupon the next trial began. Habituation trials ceased when infants' looking time (on any trial) was equal to or less than $50 \%$ of the average looking time for the first two trials. If this criterion was not met by the $12^{\text {th }}$ trial, data were excluded from the analysis.

Test Phase. The test phase consisted of two trials, in each of which two faces (the habituated face and a novel face) were presented. On each trial the paired faces were presented for 5 seconds, and the left-right position of the faces reversed between trials. 


\section{Results}

Preliminary analyses of habituation and test trials revealed no significant effects or interactions involving participant gender or view-order condition, so the data were collapsed across these factors in subsequent analyses.

Habituation trials. Habituation time (total looking time across trials) was analyzed in a 2 (participant age: $3-4$ or 8-9 months) x 3 (face race: Chinese, Malay, or Caucasian-White) x 2 (face gender: female or male) mixed ANOVA with face gender as a repeated measure. This yielded a significant effect of face gender, $F(1,60)=$ $20.668, p<.001, \eta^{2}=.256$, in which male faces were habituated to more quickly $(M=$ 35.1s) than females faces $(M=47.25 \mathrm{~s})$. A significant face gender $\mathrm{x}$ age interaction was also found, $F(1,60)=4.308, p=.042, \eta^{2}=.067$ (see Table 1). Simple main effects showed that although male faces were habituated to more quickly than female faces in both 3- to 4- and 8- to 9-month-olds ( $p \leq .025), 3$ - to 4-month-olds habituated more slowly than 8 - to 9-month-olds to female faces, $t(64)=-2.172, p=.034$, but there was no age effect for male faces, $t(64)=-.393, p=.696$. There were no main effects of face race, nor were there any interactions.

Table 1

Total fixation time (standard deviation) on habituation trials for female versus male faces in 4- and 9-month-olds

\begin{tabular}{lcc}
\hline Age & Female faces & Male faces \\
\hline 4-month-olds & $53.42(3.72)$ & $35.72(2.78)$ \\
9-month-olds & $41.08(3.48)$ & $34.47(2.6)$ \\
\hline
\end{tabular}

Test trials. The proportions of time spent looking at the novel stimulus (novelty-preference: see Figure 2) were averaged from both trials of the test phase and 
analyzed in a 2 (age: 3-4 or 8-9 months) x 3 (face race: Chinese, Malay, or CaucasianWhite) x 2 (face gender: female or male) mixed ANOVA with face gender as a within factor. There were no significant main effects. But, importantly in relation to our prediction, there was a significant 3-way interaction between age $\mathrm{x}$ face race $\mathrm{x}$ face gender, $F(2,69)=7.453, p<.001, \eta^{2}=.178$. To investigate this interaction, separate analyses were carried out for each age group.

The data for 3- to 4-month-olds showed a significant interaction between face race $\mathrm{x}$ face gender, $F(2,34)=5.761, p=.007, \eta^{2}=.253$. Simple main effects yielded face gender differences for Caucasian-White faces, $t(10)=2.342, p=.041$ : infants showed better recognition (reflected in larger novelty preference scores) for Caucasian-White female faces $(M=68.17)$ than Caucasian-White male faces $(M=$ 40.87). In contrast, simple main effects did not show any face gender differences for Chinese faces, $t(12)=-1.027, p=.325$, or Malay faces, $t(12)=-1.112, p=.288$. Simple main effects yielded face race differences for female faces, $F(2,34)=5.608$, $p=.008$, but not for male faces, $F(2,34)=1.849, p=.173$. Post-hoc Bonferonni comparison on female faces showed novelty-preference for Caucasian-White female faces to be significantly higher than novelty-preference for Chinese $(p=.03)$ and Malay $(p=.009)$ female faces.

In contrast, the face race $\mathrm{x}$ face gender interaction was not significant for 8- to 9-month-olds, $F(2,35)=1.722, p=.193, \eta^{2}=.090$. Instead, there was a significant main effect of face race, $F(2,35)=3.368, p=.046, \eta^{2}=.161$. Post-hoc Tukey tests revealed significant differences between Chinese and Caucasian-White faces ( $p=$ $.049)$, but not between Chinese and Malay faces $(p=.886)$, or Malay and CaucasianWhite faces $(p=.143)$. 

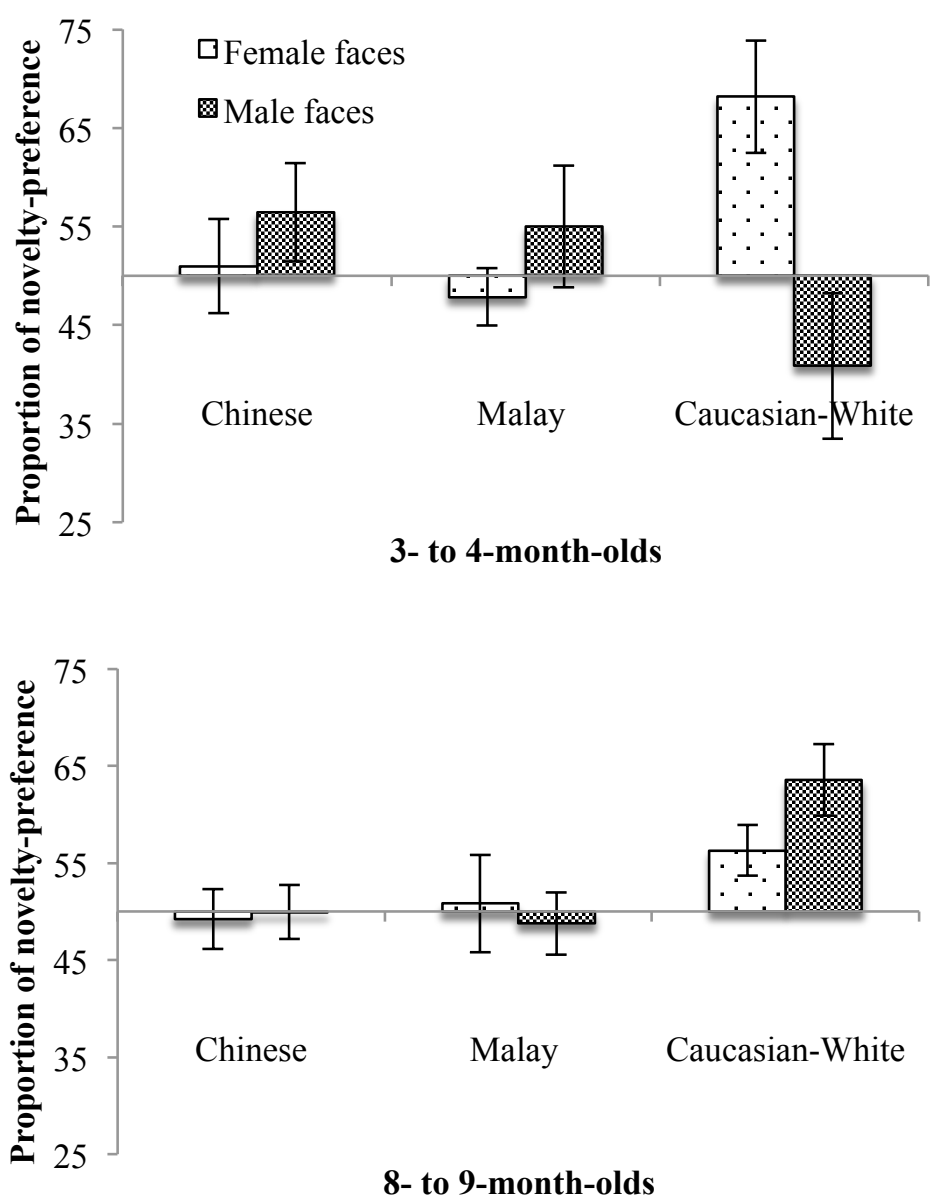

Figure 2. Proportion of novelty-preference as a function of participant age, face race, and face gender. Note. 'Rising' bars indicate novelty-preference.

To further investigate novelty-preferences within each age group, we conducted a series of two-tailed $t$-tests to determine whether the time spent looking at novel stimuli differed from chance (50\%). Three- to four-month-olds demonstrated a significant departure from chance showing novelty-preference for female CaucasianWhite faces $(p=.009)$, and 8- to 9-month-olds did for both female $(p=.033)$ and male $(p=.004)$ Caucasian-White faces (see Figure 2$)$. The own-race novelty preference for male Caucasian-White faces was at chance level for 3- to 4-month-olds $(p=.243)$. And the other-race novelty-preferences were at chance level for both 3- to 4- and 8- to 9-month-olds (Chinese faces, $p \geq .219$; Malay faces $p \geq .434$ ). 


\section{Discussion}

Two major findings emerged from this investigation. Firstly, accuracy of face recognition at 3-4 months depends on both face gender and face race. At this age, infants showed face recognition only for female own-race faces. Secondly, at 8-9 months, face recognition is simply dependent on race and is no longer dependent on gender. Infants are better at recognizing both female and male own-race faces compared with faces from other, less experienced races.

The finding with the younger age group is consistent with the findings of Sangrigoli and de Schonen (2004), and Hayden et al. (2007) that younger (3-monthold) Caucasian-White infants showed an ORE for female faces. This agreement in results is in keeping with our suggestion that face specialization should be evident first with female, own-race faces and would be most likely to be evident when using cropped faces that required infants to process configurational facial information. In contrast, it seems likely that studies that found no evidence of early specialization for own-race or female faces at 3 to 4 months (Ferguson et al., 2007; Kelly et al., 2007b, 2009) arose because they were able to rely on external detail such as hairstyle. Thus, it is possible that their work tapped into a low-level process that may not be subject to the same specialization as a result of experience. This conclusion is in keeping with previous work showing better face recognition from the external features than the internal ones in young infants (e.g., Pascalis et al., 1995; Turati et al., 2006).

In contrast, 8- to 9-month-olds showed a general ORE that was not specific to gender, which is consistent with previous discrimination studies on infants (e.g., Kelly et al., 2007b, 2009). It is likely that this developmental change relates to infants' changing face experience. In most instances, young infants have more exposure to adult female faces than they have to adult male faces (Ramsey, Langlois, \& Marti, 
2005; Ramsey-Rennels \& Langlois, 2006; Rennels \& Davis, 2008). However, by 5 months, there is a threefold increase in experience with male strangers (see Rennels \& Davis, 2008, p.671), which may explain the extension of specialization (and hence the ORE) to male faces. This is consistent with exposure training studies to reduce the ORE in infants (e.g., Anzures et al., 2012; Heron-Delaney et al., 2011; Sangrigoli \& de Schonen, 2004). It can be argued that the development of the ORE happen first in the case of primary caregivers' gender faces, and later with other gender, not necessarily due to differential contact but quality of contact (e.g., forming an attachment relationship, Scherf \& Scott, 2012)

A preference for own-race female faces emerges at 3 months for infants with predominantly own-race experience (Bar-Haim et al., 2006) and infants with female primary caregivers (Quinn et al., 2002; 2008). It is tempting to speculate that the emergence of ORE with female faces and parallel emergence of female face preference are linked. A newborn tendency to orient to face stimuli in general (see Johnson, Ellis, \& Morton, 1991, CONSPEC) may be replaced to a tendency to orient to and learn about the face(s) matching the gender of the most frequently experienced faces. Thus, by 3 months, infants show both a spontaneous preference to orient to female faces and face specialization for female faces reflected in emergence of the ORE for that gender alone. Some months later, however, likely through increased experience with male faces, the tendency to orient to male faces increases so that the gender preference is eliminated and face specialization (and the ORE) becomes general across gender. In Experiment 2, to test this potential link, we examined 4- and 9-month-old Caucasian-White infants' spontaneous preferences for own-race female versus male faces. Our prediction was that there would be a female face preference at 4 months but no gender preference at 9 months. 


\section{Experiment 2}

\section{Method}

Participants. A second sample of 28 Caucasian-White infants, 14 aged 4 months ( 6 females, mean age $=129$ days: range $116-147$ days $)$ and 14 aged 9 months ( 5 females, mean age $=279$ days: range 260 - 297 days $)$ from the population described above participated in the spontaneous visual preference (VP) task. An additional five infants were excluded due to side biases $(n=2)$ and fussiness $(n=3)$. Again, all participants had female (own-race) primary caregivers.

Stimuli. A total of sixteen Caucasian-White adult faces ( 8 males and 8 females) were used as stimuli. Each of the selected 16 adults photographs were presented full-face in a frontal orientation. These faces were selected based on the same adult raters' judgments on a 7-point scale of attractiveness with 7 being 'extremely attractive' and 1 being 'extremely unattractive from a pool of 30 Caucasian-White faces. Faces that were judged as average were selected as stimuli for this experiment. We used this rating scale because previous research on face preference only focused on adult raters' attractiveness judgment (e.g., Quinn et al., 2002). The female faces had a mean rating of $4.25(\mathrm{SD}=.42)$ and the male faces had a mean rating of $4.36(\mathrm{SD}=.62)$.

Procedure. Infants viewed six 10 sec. preference trials, each of which paired a male face with a female face. Each trial presented pairings of different faces randomly ordered. The left-right positioning of the two genders was counterbalanced across infants. Each trial began with a presentation of the attention-getter until infants directed their gaze to the screen. 


\section{Results and Discussion}

To determine whether infants showed a spontaneous visual preference for one of the two face genders, preference scores for female faces were calculated for each infant (Quinn et al., 2002; Quinn et al., 2008). The total length of time looking at the female faces was divided by the total time spent looking at both female and male stimuli. Four-month-olds' mean preference for female faces $(M=57.8 \%$; $\mathrm{SD}=9 \%)$ was reliably different from chance, $t(13)=3.255, p=.006, \eta^{2}=.67$, and 12 out of 14 infants had preferences above $50 \%$, binomial probability, $p=.01$. By contrast, 9 month-olds' preference for female over male faces was not significantly different from chance, $M=51.38 \%(\mathrm{SD}=7 \%), t(13)=.721, p=.484, \eta^{2}=.196$, and only 6 of 14 infants had preferences above 50\% (binomial probability, $p=.79$ ). Moreover, the female preference mean scores at 4 and 9 months were reliably different, $t(26)=$ $2.09, p=.047, \eta^{2}=.379$.

Replicating the Quinn et al. (2002) finding with 3-month-olds, our 4-monthold infants showed a spontaneous preference for female over male own-race faces when infants had female primary caregivers. Importantly, however, this preference was absent in 9-month-olds.

\section{General Discussion}

In Experiment 1, younger infants did not show face discrimination for other races, but perform better than chance for own-race female faces. In addition, this female recognition advantage is likely linked to preference for female faces, particularly when infants' primary caregiver is female. Of equal importance, 9-monthold infants in the current study showed recognition advantage for own-race faces regardless of gender and the lack of a female recognition advantage may be linked to the absence of gender preference in the older age group. 
It would seem that our results from both the novelty-preference and the spontaneous-preference study are consistent with the modifiable multidimensional face-space model (Valentine, 1991) according to which faces are encoded relative to the central tendency (or face prototype), which is based on distinctiveness and experience of the encoded face. From birth, infants show a tendency to orient toward faces, preferring prototype (attractive) faces. This initial prototype is general across gender and race (and possibly to an extent even species). However, this orienting process leads to specialization in the face space, such that both orienting preference and processing advantages are shifted towards the face type most frequently encountered, typically female faces. Furthermore, as Scherf and Scott (2012) noted, the essential developmental task of forming attachment relationships with caregivers involves seeking proximity and perceptually discriminating the primary caregivers from all others, making female own-race faces the most socially and emotionally relevant of all faces in young infants' world (e.g., Fagan, 1976; Ward, 1998; Walton et al., 1992). Later in development, in part because experience of male faces increases, both the orienting preference and the processing advantage (and hence the ORE) become general to male and female faces. According to an attachment-based account, as infants establish additional attachment relationships with other individuals (e.g., male caregivers), their representational space for faces will self-organise to reflect the perceptual characteristics of these other individuals. Therefore, instead of suggesting a purely experienced-based explanation in understanding the ORE of face processing advantages in infants, it is likely that social factors such as attachment relationships may influence changes in how infants attend and process faces.

An important avenue for future research is the investigation of own- versus other-race face discrimination in infants raised in a heterogeneous versus 
homogeneous environments. Some work already exists on face recognition in infants reared in heterogeneous environments. For example, in their investigation of 3month-olds' preferences for own- versus other-race faces, Bar-Haim et al. (2006) found that Ethiopian African 3-month-olds living in a heterogeneous housing unit for new immigrants in Israel showed no preference for either African or Caucasian-White faces. In terms of face discrimination, a recent study by Gaither, Pauker, \& Johnson (2012) showed that 3-month-old Caucasian-White and Asian infants raised in a heterogeneous environment did not indicate any novelty-preference for either Caucasian-White or Asian faces. Specifically, these infants did not discriminate between exemplars of own-race and other-race female faces. The authors suggest that the ORE may not develop as early for infants who grow up in a racially diverse environment. Direct comparison of face recognition by infants growing up in homogeneous and heterogeneous environments will add to our understanding of the effects of differing experience on face recognition.

In addition, because the present finding with 3- to 4-month-olds and the previous findings with 3-month-olds (Hayden et al., 2007; Sangrigoli \& de Schonen, 2004) showed an ORE when external facial information was controlled, the next important question is whether an initial race-general perceptual ability can be found in infants younger than 3-months when using the same cropped faces.

In conclusion, the current study is the first to systematically investigate the interaction between face race and face gender within the first year of life. Our main finding was that ORE at 3 to 4 months of age is limited to female faces and coincides with infants' predominant experience with female own-race faces during that period. A plausible basis for these results is that the early emergence of preference and ORE for female faces is related to infants' experience of the primary caregiver, and it is 
likely that both quantity and quality of contact (the latter likely relating to attachment) contribute to this gender effect and its subsequent disappearance. Investigation of the relative contribution of quantity and quality of contact is beyond the scope of the present paper, but should be an important question for future work. 


\section{References}

1.Anzures, G., Wheeler, A., Quinn, P. C., Pascalis, O., Slater, A. M., HeronDelaney, M., et al. (2012). Brief daily exposures to Asian females reverses perceptual narrowing for Asian faces in Caucasian infants. Journal of Experimental Child Psychology, 112(4), 484-495. doi:10.1016/j.jecp.2012.04.005

2. Bar-Haim, Y., Ziv, T., Lamy, D., \& Hodes, R. (2006). Nature and nurture in own-race face processing. Psychological Science, 17(2), 159-163.

3.Barrera, M. E., \& Maurer, D. (1981). Recognition of mother's photographed face by the three-month-old infant. Child Development, 52(2), 714-716.

4. Bartrip, J., Morton, J., \& de Schonen, S. (2001). Responses to mother's face in 3-week to 5-month-old infants. British Journal of Developmental Psychology, 19, 219-232.

5. Bruce, V., \& Young, A. (1986). Understanding face recognition. British Journal of Psychology, 77, 305-327.

6. Bruce, V., Henderson, Z., Greenwood, K., Hancock, P. J. B., Burton, A. M., \& Miller, P. (1999). Verification of face identities from images captured on video. Journal of Experimental Psychology: Applied, 5(4), 339-360.

7. Bushnell, I. W. R., Sai, F., \& Mullin, J. T. (1989). Neonatal recognition of the mother's face. British Journal of Developmental Pscyhology, 7, 3-15.

8. Cohen, L.B., Atkinson, D.J., \& Chaput, H. H. (2004). Habit X: A new program for obtaining and organizing data in infant perception and cognition studies (Version 1.0). Austin: University of Texas.

9. Fagan, J. F. (1976). Infants' recognition of invariant features of faces. Child Development, 47, 627-638. 
10. Ferguson, K., Kulkofsky, S., Cashon, C., \& Casasola, M. (2009). The development of specialized processing of own-race faces in infancy. Infancy, 14(3), 263-284. doi:10.1080/15250000902839369

11. Gaither, S. E., Pauker, K., \& Johnson, S. P. (2012). Biracial and monoracial infant own-race face perception: an eye tracking study. Developmental Science, 15(6), 775-782. doi:10.1111/j.1467-7687.2012.01170.x

12. Hainline, L. (1978). Developmental changes in visual scanning of face and nonface patterns by infants. Journal of Experimental Child Psychology, 25, $90-115$.

13. Haith, M. M., Bergman, T., \& Moore, M. J. (1977). Eye Contact and Face Scanning in Early Infancy. Science, 198(4319), 853-855.

14. Hayden, A., Bhatt, R. S., Joseph, J. E., \& Tanaka, J. W. (2007). The otherrace effect in infancy: Evidence using a morphing technique. Infancy, 12(1), 95-104.

15. Heron-Delaney, M., Anzures, G., Herbert, J. S., Quinn, P. C., Slater, A. M., Tanaka, J. W., et al. (2011). Perceptual training prevents the emergence of the other-race effect during infancy. PLoS ONE, 6(5), e19858. doi:10.1371/journal.pone.0019858.t003

16. Johnson, M., Ellis, M., \& Morton, J. (1991). Newborns’ preferential tracking of face-like stimuli and its subsequent decline. Cognition, 40 1-19.

17. Kelly, D. J., Liu, S., Ge, L., Quinn, P. C., Slater, A. M., Lee, K., Liu, Q., \& Pascalis, O. (2007a). Cross-race preferences for same-race faces extend beyond the African versus Caucasian contrast in 3-month-old infants. Infancy, $11(1), 87-95$.

18. Kelly, D. J., Liu, S., Lee, K., Quinn, P. C., Pascalis, O., Slater, A. M., \& Ge, 
L. (2009). Development of the other-race effect during infancy: Evidence toward universality? Journal of Experimental Child Psychology, 104(1), 105114. doi:10.1016/j.jecp.2009.01.006

19. Kelly, D. J., Quinn, P. C., Slater, A. M., Lee, K., Ge, L., \& Pascalis, O. (2007b). The other-race effect develops during infancy. Psychological Science, 18(12), 1084-1089.

20. Kelly, D. J., Quinn, P.C., Slater, A. M., Lee, K., Gibson, A., Smith, M., Ge, L., \& Pascalis, O. (2005). Three-month-olds but not newborns prefer own-race faces. Developmental Science, 8(6), F31-F36.

21. Maurer, D., \& Salapatek, P. (1976). Developmental changes in the scanning of faces by young infants. Child Development, 47(2), 523-527.

22. Meissner, C. A., \& Brigham, J. C. (2001). Thirty years of investigating the own-race bias in memory for faces: A meta-analytic review. Psychology, Public Policy, and Law, 7(1), 3-35. doi:10.1037//1076-8971.7.1.

23. Nelson, C. A. (2001). The development and neural bases of face recognition. Infant and Child Development, 10(1-2), 3-18. doi:10.1002/icd.239

24. Pascalis, O., de Schonen, S., Morton, J., Deruelle, C., \& Fabre-Grenet, M. (1995). Mother's face recognition by neonates: A replication and an extension. Infant Behavior \& Development, 18, 79-85.

25. Quinn, P. C., \& Tanaka, J. W. (2007). Early development of perceptual expertise: Within basic-level categorization experience facilitates the formation of subordinate-level category representations in 6- to 7-month-old infants. Memory \& Cognition, 35(6), 1422-1431.

26. Quinn, P. C., Uttley, L., Lee, K., Gibson, A., Smith, M., Slater, A. M., \& Pascalis, O. (2008). Infant preference for female faces occurs for same- but 
not other-race faces. Journal of Neuropsychology, 2(1), 15-26.

doi:10.1348/174866407X231029

27. Quinn, P. C., Yahr, J., Kuhn, A., Slater, A. M., \& Pascalis, O. (2002). Representation of the gender of human faces by infants: A preference for female. Perception, 31(9), 1109-1121. doi:10.1068/p3331

28. Ramsey, J. L., Langlois, J. H., \& Marti, N. C. (2005). Infant categorization of faces: Ladies first. Developmental Review, 25(2), 212-246.

doi:10.1016/j.dr.2005.01.001

29. Ramsey-Rennels, J. L., \& Langlois, J. H. (2006). Infants' differential processing of female and male faces. Current Directions in Psychological Science, 15(2), 59-62.

30. Rennels, J. L., \& Davis, R. E. (2008). Facial experience during the first year. Infant Behavior \& Development, 31(4), 665-678. doi:10.1016/j.infbeh.2008.04.009

31. Rose, S. A., Jankowski, J. J., \& Feldman, J. F. (2008). The inversion effect in infancy: The role of internal and external features. Infant Behavior \& Development, 31(3), 470-480. doi:10.1016/j.infbeh.2007.12.015

32. Sangrigoli, S., \& de Schonen, S. (2004). Recognition of own-race and otherrace faces by three-month-old infants. Journal of Child Psychology and Psychiatry, 45(7), 1219-1227. doi:10.1111/j.1469-7610.2004.00319.x

33. Scherf, K. S., \& Scott, L. S. (2012). Connecting developmental trajectories: Biases in face processing from infancy to adulthood. Developmental Psychobiology, 54, 643-663.

34. Slater, A. M., von der Schulenburgh, C., Brown, E., Badenoch, M., Butterworth, G., Parsons, S., \& Samuels, C. (1998). Newborn infants prefer 
attractive faces. Infant Behavior and Development, 21, 345-354.

35. Turati, C., Cassia, V. M., Simion, F., \& Leo, I. (2006). Newborns' Face Recognition: Role of Inner and Outer Facial Features. Child Development, $77(2), 297-311$.

36. Ward, C. D. (1998). The role of multisensory information in infants' recognition of their fathers. Doctoral Dissertation, Virginia Polytechnic Institute and State University.

37. Walton, G. E., Bower, N. J. A., \& Bower, T. G. R. (1992). Recognition of familiar faces by newborns. Infant Behavior and Development, 15, 265-269.

38. Valentine, T. (1991). A unified account of the effects of distinctiveness, inversion, and race in face recognition. The Quarterly Journal of Experimental Psychology Section A, 43(2), 161-204. doi:10.1080/14640749108400966 